\title{
Bayesian Potentials and Information Structures: Team Decision Problems Revisited*
}

\author{
Takashi $\mathrm{Ui}^{\dagger}$ \\ Faculty of Economics \\ Yokohama National University \\ oui@ynu.ac.jp \\ First Draft: September 2002 \\ This Version: September 2008
}

${ }^{*}$ I thank an anonymous referee for detailed comments and suggestions. I also thank seminar participants at Tohoku University, Keio University, Hitotsubashi University, and Annual Meeting of Japanese Economic Association at Oita University for valuable comments and discussions. I acknowledge financial support by MEXT, Grant-in-Aid for Scientific Research.

$\dagger^{\dagger}$ Faculty of Economics, Yokohama National University, 79-3 Tokiwadai, Hodogaya-ku, Yokohama 240-8501, Japan. Phone: (81)-45-339-3531. Fax: (81)-45-339-3574. 


\begin{abstract}
This paper proposes the use of Bayesian potential games as models of informationally decentralized organizations. Applying techniques in team decision problems by Radner (1962, Ann. Math. Statist. 33, 857-881), this paper characterizes Bayesian Nash equilibria in terms of Bayesian potentials and demonstrates by examples that Bayesian potentials are useful tools in studying the efficient use of information in organizations.
\end{abstract}

JEL classification: C72, D82.

Keywords: team; information structure; Bayesian potential game; best response equivalence. 


\section{Introduction}

In an organization, individuals typically differ in at least three important respects: they control different actions; they base their decisions on different information; they have different goals. Marshak and Radner (1972) argued that some interesting aspects of organizations mainly concern differences of actions and information only and developed team theory (cf. Marshak, 1955; Radner, 1961; Radner, 1962). A team is a model of an organization in which individuals with a common goal control different actions based upon different information. The common goal assumption is a technical requirement which makes the model mathematically tractable. From the viewpoint of game theory, a team is a Bayesian identical interest game where every player has the identical payoff function. Marshak and Radner (1972) characterized an optimal decision function, which is a Pareto efficient Bayesian Nash equilibrium, and studied the efficient use of information in organizations.

Following Marshak and Radner (1972), many studies on organizations have taken the team theoretical approach. Early works include Groves and Radner (1972) and Arrow and Radner (1979). After more than a decade of limited use (when principal-agent theory played a dominant role in economic theory of organizations), team theory has been experiencing a renewed interest. Several authors have applied it to problems in economic theory of organizations that do not seem to find a satisfactory answer within the principal-agent framework. Examples include Crémer (1980, 1990, 1993), Arrow (1985), Aoki (1986, 1995), Itoh (1987), Geanakoplos and Milgrom (1991), Bolton and Dewatripont (1994), Prat (1996, 2002), and Qian et al. (2000). ${ }^{1}$

In some cases, however, the common goal assumption of team theory seems too strong because of the following reason: more information is always more favorable when individuals have a common goal, whereas more information can be less favorable when individuals have different goals as demonstrated by Levine and Ponssard (1977), Bassan et al. (1997), and Morris and Shin (2002), among others, in game theoretical frameworks. ${ }^{2}$ This suggests that the efficient use of information in teams may not be so in organizations other than teams. It will be useful if we have a model of an organization such that it is as mathematically tractable as a team and it does not require the common goal assumption.

Motivated by the above observation, we propose the use of a Bayesian potential game (Monderer and Shapley, 1996; van Heumen et al., 1996) as a model of an organization. That is, we consider a model of an organization in which individuals with different goals control different actions based upon different information such that it possesses a potential function. A game is a potential game if there exists a potential function defined on the action space with the property that the change in any player's payoff function from switching between any two of his actions (holding other players' actions fixed) is equal to the change in the potential function (Monderer and Shapley, 1996). In a potential game, the set of Nash equilibria is the same as that of an identical interest game such that every player's payoff function is identical to the potential function. A Bayesian game is said to be a Bayesian potential game if, for each state, a fictitious game composed of a payoff function profile at the state is a potential game (van Heumen et al., 1996). In a Bayesian potential game, the set of Bayesian Nash equilibria is the same as that of a Bayesian identical interest game, or a team, such that every player's payoff function is identical to the potential function at every state.

\footnotetext{
${ }^{1}$ See also Radner (1992) and Van Zandt (1999).

${ }^{2}$ Neyman (1991), Gossner (2000), and Bassan et al. (2003) discussed sufficient conditions under which more information is always more favorable.
} 
Once we have constructed a model of an organization using a Bayesian potential game, we can characterize its Bayesian Nash equilibria using techniques of team theory because the set of Bayesian Nash equilibria is the same as that of the team. Using the results of Radner (1962), we argue that if a potential function is concave at every state, then any Bayesian Nash equilibrium must be a Pareto efficient Bayesian Nash equilibrium of the team, and that if a potential function is concave and quadratic at every state and private signals are normally distributed, then the Bayesian Nash equilibrium is linear in private signals. In the latter case, we can obtain the Bayesian Nash equilibrium in a closed form.

This argument applies to a larger class of Bayesian games, which we call Bayesian best-response potential games. A Bayesian game is said to be a Bayesian best-response potential game if interim best-response correspondences of the Bayesian game coincide with those of a Bayesian identical interest game, or a team. ${ }^{3}$ In a Bayesian best-response potential game, the set of Bayesian Nash equilibria is the same as that of the team, and thus we can use techniques of team theory. We show that a class of Bayesian best-response potential games are strictly larger than that of (weighted) Bayesian potential games.

We demonstrate by examples how our approach works in studying the efficient use of information. The examples employ a parametrized class of two-player, symmetric, quadratic Bayesian potential games with normally distributed private signals. First, we provide conditions for the parameters under which most precise information is most favorable, and under which most precise information is least favorable. Next, we examine the result of Crémer (1990) comparing two information structures in teams: shared knowledge and diversified knowledge. ${ }^{4}$ Shared knowledge refers to an information structure in which every individual has the same information and diversified knowledge refers to an information structure in which every individual has conditionally independent information. Crémer (1990) showed that shared knowledge is better than diversified knowledge if and only if a team exhibits strategic complementarity. We show that this is true for organizations other than teams. Finally, we examine the result of Morris and Shin (2002) considering welfare effects of public information in Bayesian coordination games with strategic complementarity. They demonstrated that more private information always results in more efficiency whereas more public information may result in less efficiency depending upon the accuracy of private information. We show that this is true for a larger class of Bayesian games with a slightly different setup.

Basar and Ho (1974) was the first to use the results of Radner (1962) for Bayesian games. They considered two-player quadratic Bayesian games with normally distributed private signals, which include Bayesian duopoly games with linear demand functions, and demonstrated that the calculation of Bayesian Nash equilibria is reduced to that in teams. Following Basar and Ho (1974), many papers on information sharing in Bayesian oligopoly games with linear demand functions have took the same approach: see Clark (1983), Gal-Or (1985, 1986), Vives (1984, 1988), Raith (1996) among others. It is worth pointing out that Bayesian games studied in the above papers form a special class of Bayesian potential games because oligopoly games with linear demand functions are potential games as shown by Slade (1994). In contrast, this paper uses the results of Radner (1962) for a larger class of Bayesian best-response potential games, generalizing the approach taken by the above papers for broader purposes. A recent paper by Angeletos and Pavan (2007) study the efficient use of information using continuum-of-player quadratic Bayesian

\footnotetext{
${ }^{3} \mathrm{~A}$ best-response potential game with complete information is introduced by Morris and Ui (2004).

${ }^{4}$ We adopt the terms "shared knowledge" and "diversified knowledge" from Prat (1996) who generalized the result of Crémer (1990).
} 
potential games with normally distributed private signals. A finite-player version of their Bayesian games also form a special class of Bayesian potential games we study.

Let us conclude this introduction with an outline of this paper. Section 2 reviews potential games as a preparation for studying Bayesian potential games. Section 3 introduces Bayesian potential games. Section 4 characterizes Bayesian Nash equilibria using the results of Radner (1962). Section 5 demonstrates by examples how our approach works. Section 6 discusses the extension to Bayesian best-response potential games.

\section{Potential games}

A game (with complete information) consists of a set of players $N=\{1, \ldots, n\}$, a set of actions $A_{i}$ for $i \in N$, and a payoff function $g_{i}: A \rightarrow \mathbb{R}$ for $i \in N$ where $A=\prod_{i \in N} A_{i}$. We write $A_{-i}=\prod_{j \neq i} A_{j}$. We simply denote a game by $\mathbf{g}=\left(g_{i}\right)_{i \in N}$. In this paper, it will be assumed that $A_{i} \subseteq \mathbb{R}$ is an interval for each $i \in N$. We regard $a \in A$ as a column vector in $\mathbb{R}^{n}$.

Potential games and weighted potential games are formally defined by Monderer and Shapley (1996).

Definition 1 A game $\mathbf{g}$ is a weighted potential game if there exists a weighted potential function $f: A \rightarrow \mathbb{R}$ with a constant $w_{i}>0$ such that

$$
g_{i}\left(a_{i}, a_{-i}\right)-g_{i}\left(a_{i}^{\prime}, a_{-i}\right)=w_{i}\left(f\left(a_{i}, a_{-i}\right)-f\left(a_{i}^{\prime}, a_{-i}\right)\right)
$$

for all $a_{i}, a_{i}^{\prime} \in A_{i}, a_{-i} \in A_{-i}$, and $i \in N$. If $w_{i}=1$ for all $i \in N$, $\mathbf{g}$ is called a potential game and $f$ is called a potential function.

For example, consider $\mathbf{g}$ such that

$$
g_{i}(a)=\frac{v(a)}{n}-c_{i}\left(a_{i}\right)
$$

where $v: A \rightarrow \mathbb{R}$ and $c_{i}: A_{i} \rightarrow \mathbb{R}$ for $i \in N$. We regard $\mathbf{g}$ as a simple model of a firm where $v(a)$ is a total value produced by the firm, $v(a) / n$ is each player's share of the value, and $c_{i}\left(a_{i}\right)$ is a private cost of player $i$ choosing $a_{i} \in A_{i}$. This game is a potential game with a potential function $f(a)=v(a) / n-\sum_{i} c_{i}\left(a_{i}\right)$ because

$$
\begin{aligned}
g_{i}\left(a_{i}, a_{-i}\right)-g_{i}\left(a_{i}^{\prime}, a_{-i}\right) & =f\left(a_{i}, a_{-i}\right)-f\left(a_{i}^{\prime}, a_{-i}\right) \\
& =\frac{v\left(a_{i}, a_{-i}\right)}{n}-\frac{v\left(a_{i}^{\prime}, a_{-i}\right)}{n}-c_{i}\left(a_{i}\right)+c_{i}\left(a_{i}^{\prime}\right) .
\end{aligned}
$$

Hart and Moore (1990) considered a model of a firm which generalizes the above game where each player's share of the value is not necessarily equal and determined by the "Shapley value" rule. As shown by Ui (2000), the model of Hart and Moore (1990) is also a potential game.

A weighted potential function $f$ is a useful tool in finding Nash equilibria. To see this, observe that

$$
\int\left(g_{i}\left(a_{i}, a_{-i}\right)-g_{i}\left(a_{i}^{\prime}, a_{-i}\right)\right) d \lambda_{i}\left(a_{-i}\right)=w_{i} \int\left(f\left(a_{i}, a_{-i}\right)-f\left(a_{i}^{\prime}, a_{-i}\right)\right) d \lambda_{i}\left(a_{-i}\right)
$$

for all $a_{i}, a_{i}^{\prime} \in A_{i}$ where $\lambda_{i}$ is a probability measure on $A_{-i}$. Thus,

$$
\arg \max _{a_{i} \in A_{i}} \int g_{i}\left(a_{i}, a_{-i}\right) d \lambda_{i}\left(a_{-i}\right)=\arg \max _{a_{i} \in A_{i}} \int f\left(a_{i}, a_{-i}\right) d \lambda_{i}\left(a_{-i}\right),
$$

which implies the following lemma. 
Lemma 1 (Monderer and Shapley, 1996) For a weighted potential game $\mathrm{g}$ with a weighted potential function $f$, let $\mathbf{f}$ be an identical interest game such that $\mathbf{f}=\left(f_{i}\right)_{i \in N}$ and $f_{i}=f$ for all $i \in N$. Then, the set of Nash equilibria of $\mathbf{g}$ coincides with that of $\mathbf{f}$. Especially, a potential maximizer $a^{*} \in \arg \max _{a \in A} f(a)$ is a Nash equilibrium of $\mathbf{g}$ if it exists.

The following two lemmas contain necessary and sufficient conditions for potential games.

Lemma 2 (Monderer and Shapley, 1996) Suppose that $g_{i}$ is twice continuously differentiable for each $i \in N$. A game $\mathbf{g}$ is a potential game if and only if

$$
\frac{\partial^{2} g_{i}}{\partial a_{i} \partial a_{j}}=\frac{\partial^{2} g_{j}}{\partial a_{i} \partial a_{j}}
$$

for all $i, j \in N$. Moreover, for an arbitrary fixed $b \in A$, a potential function is given by

$$
f(a)=\sum_{i \in N} \int_{0}^{1} \frac{\partial g_{i}}{\partial a_{i}}(x(t)) \frac{d x_{i}(t)}{d t} d t
$$

where $x:[0,1] \rightarrow A$ is a piecewise continuously differentiable path in $A$ that connects $b$ to a, i.e., $x(0)=a$ and $x(1)=b$.

Lemma 3 (Ui, 2000) For a subset of players $S \subseteq N$, let $A_{S}=\prod_{i \in S} A_{i}$ be a restricted action space with a generic element $a_{S}=\left(a_{i}\right)_{i \in S}$. A game $\mathbf{g}$ is a potential game if and only if there exists a collection of functions $\left\{\Phi_{S}: A_{S} \rightarrow \mathbb{R}\right\}_{S \in 2^{N}}$ such that

$$
g_{i}(a)=\sum_{S \subseteq N, i \in S} \Phi_{S}\left(a_{S}\right)
$$

for all $a \in A$ and $i \in N$. A potential function is given by

$$
f(a)=\sum_{S \subseteq N} \Phi_{S}\left(a_{S}\right)
$$

Consider a game with quadratic payoff functions such that

$$
g_{i}(a)=-q_{i i} a_{i}^{2}-2 \sum_{j \neq i} q_{i j} a_{i} a_{j}+2 \theta_{i} a_{i}+h_{i}\left(a_{-i}\right)
$$

where $q_{i i}, q_{i j}$, and $\theta_{i}$ are constants and $h_{i}: A_{-i} \rightarrow \mathbb{R}$ for $i \in N$. By Lemma 2, $\mathbf{g}$ is a potential game if and only if

$$
\frac{\partial^{2} g_{i}}{\partial a_{i} \partial a_{j}}=-2 q_{i j}=-2 q_{j i}=\frac{\partial^{2} g_{j}}{\partial a_{i} \partial a_{j}} \text { for all } i \neq j .
$$

Let us find a potential function using Lemma $3{ }^{5}$ For this purpose, we can assume $h_{i}\left(a_{-i}\right)=0$ without loss of generality because, by the definition of potential games, if $f$ is a potential function of $\mathbf{g}$ then it is also a potential function of $\mathbf{g}^{\prime}$ with $g_{i}^{\prime}(a)=g_{i}(a)+h_{i}^{\prime}\left(a_{-i}\right)$ for any $h_{i}^{\prime}: A_{-i} \rightarrow \mathbb{R}$. Assuming that $q_{i j}=q_{j i}$, let $\left\{\Phi_{S}\right\}_{S \in 2^{N}}$ be such that $\Phi_{\{i, j\}}\left(a_{\{i, j\}}\right)=-2 q_{i j} a_{i} a_{j}, \Phi_{\{i\}}\left(a_{i}\right)=-q_{i i} a_{i}^{2}+$ $2 a_{i} \theta_{i}$, and $\Phi_{S}\left(a_{S}\right)=0$ otherwise. Then,

$$
\sum_{S \subseteq N, i \in S} \Phi_{S}\left(a_{S}\right)=-q_{i i} a_{i}^{2}-2 \sum_{j \neq i} q_{i j} a_{i} a_{j}+2 \theta_{i} a_{i}=g_{i}(a)
$$

and thus the potential function is

$$
f(a)=\sum_{S \subseteq N} \Phi_{S}\left(a_{S}\right)=\sum_{\{i, j\} \subseteq N} \Phi_{\{i, j\}}\left(a_{\{i, j\}}\right)+\sum_{i \in N} \Phi_{\{i\}}\left(a_{i}\right)=-\sum_{i, j} q_{i j} a_{i} a_{j}+2 \sum_{i} \theta_{i} a_{i} .
$$

To summarize, we have the following lemma.

\footnotetext{
${ }^{5}$ We can also use Lemma 2. But it requires integrals. On the other hand, Lemma 3 requires combinatorial consideration, which is sometimes simple.
} 
Lemma 4 A game $\mathbf{g}$ of the above form is a potential game if and only if $q_{i j}=q_{j i}$ for all $i, j \in N$. A potential function $f$ is such that

$$
f(a)=-a^{\prime} Q a+2 \theta^{\prime} a
$$

where $Q=\left[q_{i j}\right]_{n \times n}$ and $\theta=\left[\theta_{1}, \ldots, \theta_{n}\right]^{\prime}$.

If $Q$ is a positive definite matrix, then $a^{*}=Q^{-1} \theta$ is the unique maximizer of $f$. In addition, it is the unique equilibrium of $\mathbf{g}$ according to Neyman (1997), who showed that a potential maximizer is a unique correlated equilibrium of a potential game if a potential function is continuously differentiable and strictly concave. We will argue analogous results for Bayesian potential games.

\section{Bayesian potential games}

A Bayesian game consists of a set of players $N=\{1, \ldots, n\}$, a set of actions $A_{i}$ for $i \in N$, a probability space $(\Omega, \mathcal{F}, P)$, a payoff function $u_{i}: A \times \Omega \rightarrow \mathbb{R}$ for $i \in N$, a measurable space $\left(Y_{i}, \mathcal{Y}_{i}\right)$ with a measurable mapping $\eta_{i}: \Omega \rightarrow Y_{i}$ for $i \in N$. We write $\mathbf{u}=\left(u_{i}\right)_{i \in N}$ and $\eta=\left(\eta_{i}\right)_{i \in N}$. Because we will fix $N, A$, and $(\Omega, \mathcal{F}, P)$ throughout the paper, we simply denote a Bayesian game by $(\mathbf{u}, \eta)$. We call $\eta$ an information structure of $(\mathbf{u}, \eta)$. It will be assumed that, for each $i \in N$, $A_{i} \subseteq \mathbb{R}$ is an interval and $u_{i}\left(\left(\cdot, a_{-i}\right), \omega\right): A_{i} \rightarrow \mathbb{R}$ is differentiable for all $a_{-i} \in A_{-i}$ and $\omega \in \Omega$.

A strategy of player $i \in N$ is a measurable mapping $\sigma_{i}: Y_{i} \rightarrow A_{i}$. Let $\Sigma_{i}$ be the set of strategies of player $i \in N$. We write $\Sigma=\prod_{i \in N} \Sigma_{i}$ and $\Sigma_{-i}=\prod_{j \neq i} \Sigma_{j}$. We sometimes omit $\omega$ in writing $\eta_{i}(\omega)$. Thus, we write $\sigma_{i}\left(\eta_{i}\right)=\sigma_{i}\left(\eta_{i}(\omega)\right)$ for $\sigma_{i} \in \Sigma_{i}, \sigma(\eta)=\left(\sigma_{i}\left(\eta_{i}(\omega)\right)\right)_{i \in N}$ for $\sigma \in \Sigma$, and $\sigma_{-i}\left(\eta_{-i}\right)=\left(\sigma_{j}\left(\eta_{j}(\omega)\right)\right)_{j \neq i}$ for $\sigma_{-i} \in \Sigma_{-i}$, all of which are regarded as random variables.

A strategy profile $\sigma \in \Sigma$ is a Bayesian Nash equilibrium if, for a.e. $y_{i} \in Y_{i}$,

$$
E\left[u_{i}(\sigma(\eta), \omega) \mid \eta_{i}(\omega)=y_{i}\right] \geq E\left[u_{i}\left(\left(a_{i}, \sigma_{-i}\left(\eta_{-i}\right)\right), \omega\right) \mid \eta_{i}(\omega)=y_{i}\right]
$$

for all $a_{i} \in A_{i}$ and $i \in N$ where $E\left[\cdot \mid \eta_{i}(\omega)=y_{i}\right]$ is a conditional expectation operator given $\eta_{i}(\omega)=y_{i}$. The first-order condition for a Bayesian Nash equilibrium is

$$
\left.\frac{\partial}{\partial a_{i}} E\left[u_{i}\left(\left(a_{i}, \sigma_{-i}\left(\eta_{-i}\right)\right), \omega\right) \mid \eta_{i}(\omega)=y_{i}\right]\right|_{a_{i}=\sigma_{i}\left(y_{i}\right)}=0
$$

for a.e. $y_{i} \in Y_{i}$ and $i \in N$. Let $U_{i}: \Sigma \rightarrow \mathbb{R}$ be the ex ante expected payoff function: $U_{i}(\sigma)=$ $E\left[u_{i}(\sigma(\eta), \omega)\right]$. A strategy profile $\sigma \in \Sigma$ is a Bayesian Nash equilibrium if and only if $U_{i}(\sigma) \geq$ $U_{i}\left(\sigma_{i}^{\prime}, \sigma_{-i}\right)$ for all $\sigma_{i}^{\prime} \in \Sigma_{i}$ and $i \in N$.

Bayesian potential games and weighted Bayesian potential games are introduced by van Heumen et al. (1996).

Definition 2 A Bayesian game $(\mathbf{u}, \eta)$ is a weighted Bayesian potential game if there exists a weighted Bayesian potential function $v: A \times \Omega \rightarrow \mathbb{R}$ with a constant $w_{i}>0$ such that, for every $\omega \in \Omega$,

$$
u_{i}\left(\left(a_{i}, a_{-i}\right), \omega\right)-u_{i}\left(\left(a_{i}^{\prime}, a_{-i}\right), \omega\right)=w_{i}\left(v\left(\left(a_{i}, a_{-i}\right), \omega\right)-v\left(\left(a_{i}^{\prime}, a_{-i}\right), \omega\right)\right)
$$

for all $a_{i}, a_{i}^{\prime} \in A_{i}, a_{-i} \in A_{-i}$, and $i \in N$. If $w_{i}=1$ for all $i \in N,(\mathbf{u}, \eta)$ is called a Bayesian potential game and $v$ is called a Bayesian potential function. 
For a weighted Bayesian potential game $(\mathbf{u}, \eta)$ with a weighted Bayesian potential function $v$, let $(\mathbf{v}, \eta)$ be a Bayesian identical interest game such that $\mathbf{v}=\left(v_{i}\right)_{i \in N}$ and $v_{i}=v$ for all $i \in N$. Observe that, by (1), for all $a_{i}, a_{i}^{\prime} \in A_{i}, \sigma_{-i} \in \Sigma_{-i}$, and $y_{i} \in Y_{i}$,

$$
\begin{aligned}
& E\left[u_{i}\left(\left(a_{i}, \sigma_{-i}\left(\eta_{-i}\right)\right), \omega\right) \mid \eta_{i}(\omega)=y_{i}\right]-E\left[u_{i}\left(\left(a_{i}^{\prime}, \sigma_{-i}\left(\eta_{-i}\right)\right), \omega\right) \mid \eta_{i}(\omega)=y_{i}\right] \\
& \quad=w_{i}\left(E\left[v\left(\left(a_{i}, \sigma_{-i}\left(\eta_{-i}\right)\right), \omega\right) \mid \eta_{i}(\omega)=y_{i}\right]-E\left[v\left(\left(a_{i}^{\prime}, \sigma_{-i}\left(\eta_{-i}\right)\right), \omega\right) \mid \eta_{i}(\omega)=y_{i}\right]\right) .
\end{aligned}
$$

Thus, the first-order condition for a Bayesian Nash equilibrium in $(\mathbf{u}, \eta)$ and that in $(\mathbf{v}, \eta)$ are the same, and so are the interim best responses:

$$
\arg \max _{a_{i} \in A_{i}} E\left[u_{i}\left(\left(a_{i}, \sigma_{-i}\left(\eta_{-i}\right)\right), \omega\right) \mid \eta_{i}(\omega)=y_{i}\right]=\arg \max _{a_{i} \in A_{i}} E\left[v\left(\left(a_{i}, \sigma_{-i}\left(\eta_{-i}\right)\right), \omega\right) \mid \eta_{i}(\omega)=y_{i}\right]
$$

Let $V: \Sigma \rightarrow \mathbb{R}$ be such that $V(\sigma)=E[v(\sigma(\eta), \omega)]$. Then, by $(1)$,

$$
U_{i}\left(\sigma_{i}, \sigma_{-i}\right)-U_{i}\left(\sigma_{i}^{\prime}, \sigma_{-i}\right)=w_{i}\left(V\left(\sigma_{i}, \sigma_{-i}\right)-V\left(\sigma_{i}^{\prime}, \sigma_{-i}\right)\right)
$$

for all $\sigma_{i}, \sigma_{i}^{\prime} \in \Sigma_{i}$ and $\sigma_{-i} \in \Sigma_{-i}$. Thus, $V$ is a weighted potential function of $(\mathbf{u}, \eta)$ in the sense of Definition 1. All of the above leads us to the following lemma.

Lemma 5 (van Heumen et al., 1996) The set of Bayesian Nash equilibria of (u, $\eta$ ) coincides with that of $(\mathbf{v}, \eta)$. Especially, a potential maximizer $\sigma^{*} \in \arg \max _{\sigma \in \Sigma} V(\sigma)$ is a Bayesian Nash equilibrium of $(\mathbf{u}, \eta)$ if it exists.

Consider a Bayesian game with quadratic payoff functions such that

$$
u_{i}(a, \omega)=-q_{i i}(\omega) a_{i}^{2}-2 \sum_{j \neq i} q_{i j}(\omega) a_{i} a_{j}+2 \theta_{i}(\omega) a_{i}+h_{i}\left(a_{-i}, \omega\right)
$$

where $q_{i j}: \Omega \rightarrow \mathbb{R}, \theta_{i}: \Omega \rightarrow \mathbb{R}$, and $h_{i}: A_{-i} \times \Omega \rightarrow \mathbb{R}$ for $i, j \in N$. The following lemma is a straightforward consequence of Lemma 4 .

Lemma 6 A Bayesian game $(\mathbf{u}, \eta)$ of the above form is a Bayesian potential game if and only if $q_{i j}(\omega)=q_{j i}(\omega)$ for all $i, j \in N$ and $\omega \in \Omega$. A Bayesian potential function $v$ is such that

$$
v(a, \omega)=-a^{\prime} Q(\omega) a+2 \theta(\omega)^{\prime} a
$$

where $Q(\omega)=\left[q_{i j}(\omega)\right]_{n \times n}$ and $\theta(\omega)=\left[\theta_{1}(\omega), \ldots, \theta_{n}(\omega)\right]^{\prime}$.

For example, if $q_{i i}(\omega)=\alpha$ and $q_{i j}(\omega)=\beta$ for all $i \neq j$ and $\omega \in \Omega$ where $\alpha, \beta \in \mathbb{R}$ are constants, then $(\mathbf{u}, \eta)$ is a Bayesian potential game with a Bayesian potential function

$$
v(a, \omega)=-\alpha \sum_{i \in N} a_{i}^{2}-\beta \sum_{i, j \in N, i \neq j} a_{i} a_{j}+2 \sum_{i \in N} \theta_{i}(\omega) a_{i} .
$$

A continuun-of-player version of this Bayesian potential game is studied by Angeletos and Pavan $(2007){ }^{6}$

\footnotetext{
${ }^{6}$ Angeletos and Pavan (2007) obtained a Bayesian Nash equilibrium without using the Bayesian potential function.
} 


\section{Team decision problems revisited}

Consider a Bayesian identical interest game $(\mathbf{v}, \eta)$ such that $v_{i}=v$ for all $i \in N$ and a weighted Bayesian potential game $(\mathbf{u}, \eta)$ with a weighted Bayesian potential function $v$. Let $V(\sigma)=$ $E[v(\sigma(\eta), \omega)]$. Radner (1962) defined $(\mathbf{v}, \eta)$ to be a team, whose origin is the work of Marshak (1955). Radner (1962) called a strategy profile a decision function of a team, a Bayesian Nash equilibrium a person-by-person maximal decision function, a strategy profile satisfying the first-order condition a stationary decision function, and a Pareto efficient Nash equilibrium a Bayes decision function. Radner (1962) discussed conditions under which every stationary decision function is Bayes. In our words, they are conditions under which a strategy profile satisfying the first-order condition in $(\mathbf{v}, \eta)$, which is also that in $(\mathbf{u}, \eta)$, maximizes $V$. This observation leads us to the following theorems, all of which are immediate consequences of those of Radner (1962).

According to Theorem 1 of Radner (1962), if a weighted Bayesian potential function $v$ is concave, then a strategy profile satisfying the first-order condition maximizes $V$. Thus, if the maximizer of $V$ is unique, it must be the unique Bayesian Nash equilibrium satisfying the firstorder condition. The theorem requires the assumption of local finiteness. We say that $V$ is locally finite at $\sigma \in \Sigma$ if $|V(\sigma)|<\infty$ and, for any $\sigma^{\prime} \in \Sigma$ with $V\left(\sigma+\sigma^{\prime}\right)<\infty$, there exist $k_{1}, \ldots, k_{n}>0$ such that $\left|V\left(\sigma_{1}+h_{1} \sigma_{1}^{\prime}, \ldots, \sigma_{n}+h_{n} \sigma_{n}^{\prime}\right)\right|<\infty$ for all $h_{1}, \ldots, h_{n}$ with $\left|h_{1}\right| \leq k_{1}, \ldots,\left|h_{n}\right| \leq k_{n}$ where $\sigma_{i}+h_{i} \sigma_{i}^{\prime} \in \Sigma_{i}$ is such that $\left(\sigma_{i}+h_{i} \sigma_{i}^{\prime}\right)\left(y_{i}\right)=\sigma_{i}\left(y_{i}\right)+h_{i} \sigma_{i}^{\prime}\left(y_{i}\right)$ for all $y_{i} \in Y_{i}$.

Theorem 1 Let $(\mathbf{u}, \eta)$ be a weighted Bayesian potential game with a weighted Bayesian potential function $v$. Suppose that

- $v(\cdot, \omega): A \rightarrow \mathbb{R}$ is concave and differentiable for a.e. $\omega \in \Omega$,

- $\sup _{\sigma^{\prime} \in \Sigma} V\left(\sigma^{\prime}\right)<\infty$.

If $\sigma \in \Sigma$ satisfies the first-order condition for a Bayesian Nash equilibrium and $V$ is locally finite at $\sigma$, then $\sigma$ maximizes $V$.

It is not easy to verify the local finiteness condition. Krainak et al. (1982) obtained an extension of Theorem 1 of Radner (1962) with a condition which is easier to verify in some cases. The following theorem is its translation to our setup with Bayesian potential games. As Krainak et al. (1982) showed, the condition in Theorem 1 is sufficient for the condition in the following theorem.

Theorem 2 Let $(\mathbf{u}, \eta)$ be a weighted Bayesian potential game with a weighted Bayesian potential function $v$. Suppose that

- $v(\cdot, \omega): A \rightarrow \mathbb{R}$ is concave and differentiable for a.e. $\omega \in \Omega$,

- $\sup _{\sigma^{\prime} \in \Sigma} V\left(\sigma^{\prime}\right)<\infty$.

If $\sigma \in \Sigma$ satisfies the first-order condition for a Bayesian Nash equilibrium and, for every $\sigma^{\prime} \in \Sigma$ such that $\left|V\left(\sigma^{\prime}\right)\right|<\infty$,

$$
E\left[\left.\frac{\partial v(a, \omega)}{\partial a_{i}}\right|_{a=\sigma(\eta)} \times\left(\sigma_{i}^{\prime}\left(\eta_{i}\right)-\sigma_{i}\left(\eta_{i}\right)\right)\right]
$$

exists for each $i \in N$, then $\sigma$ maximizes $V$. 
For example, let $(\mathbf{u}, \eta)$ be a Bayesian potential game considered in Lemma 6 and define

$$
r=\inf _{\omega \in \Omega} \min _{a \in A} \frac{a^{\prime} Q(\omega) a}{\sum_{i \in N} q_{i i}(\omega) a_{i}^{2}} .
$$

According to Theorem 4 of Radner (1962), if $Q(\omega)$ is positive definite and $r>0$, then the condition in Theorem 1 (and thus Theorem 2) is satisfied.

Theorem 3 Let $(\mathbf{u}, \eta)$ be a Bayesian potential game considered in Lemma 6 . Let $A_{i}=\mathbb{R}$ for all $i \in N$. Suppose that $Q(\omega)$ is positive definite for a.e. $\omega \in \Omega$ and $r>0$. If $\sigma \in \Sigma$ satisfies the first-order condition for a Bayesian Nash equilibrium, then $\sigma$ maximizes $V$.

Clearly, if $Q(\omega)$ is a constant positive definite matrix, we must have $r>0$. Furthermore, according to Theorem 5 of Radner (1962), the Bayesian Nash equilibrium is unique and linear in private signals.

Theorem 4 Suppose that $Q(\omega)=Q$ for all $\omega \in \Omega$ where $Q$ is a constant positive definite matrix. Suppose that $\eta_{1}, \ldots, \eta_{n}$ are vector-valued, and that $\eta_{1}(\omega), \ldots, \eta_{n}(\omega)$ and $\theta_{1}(\omega), \ldots, \theta_{n}(\omega)$ are jointly normally distributed. If $\sigma \in \Sigma$ satisfies the first-order condition for a Bayesian Nash equilibrium, then it is the unique maximizer of $V$. It solves the system of equations

$$
\sum_{j \in N} q_{i j} E\left[\sigma_{j}\left(\eta_{j}\right) \mid \eta_{i}(\omega)=y_{i}\right]=E\left[\theta_{i} \mid \eta_{i}(\omega)=y_{i}\right]
$$

for all $y_{i} \in Y_{i}$ and $i \in N$ and $\sigma_{i}$ is a linear function of $\eta_{i}$.

By the above theorem, we can obtain a Bayesian Nash equilibrium in a closed form. Let $C_{i j}$ be the covariance matrix of $\eta_{i}$ and $\eta_{j}$. Assume that $\eta_{i}$ has mean zero. Let $G_{i}$ be the covariance matrix of $\eta_{i}$ and $\theta_{i}$. In summary,

$$
\operatorname{Cov}\left[\eta_{i}, \eta_{j}\right]=C_{i j}, E\left[\eta_{i}\right]=0, \operatorname{Cov}\left[\eta_{i}, \theta_{i}\right]=G_{i}
$$

Then, by the property of multivariate normal distributions, ${ }^{7}$

$$
E\left[\theta_{i} \mid \eta_{i}(\omega)=y_{i}\right]=E\left[\theta_{i}\right]+G_{i}^{\prime} C_{i i}^{-1} y_{i}, E\left[\eta_{j} \mid \eta_{i}(\omega)=y_{i}\right]=C_{j i} C_{i i}^{-1} y_{i}
$$

Let $\sigma$ be a Bayesian Nash equilibrium such that

$$
\sigma_{i}\left(\eta_{i}\right)=b_{i}^{\prime} \eta_{i}+c_{i}
$$

for each $i \in N$. Plugging this into (2), we have

$$
\sum_{j} q_{i j}\left(b_{j}^{\prime} C_{j i} C_{i i}^{-1} y_{i}+c_{j}\right)=E\left[\theta_{i}\right]+G_{i}^{\prime} C_{i i}^{-1} y_{i}
$$

for all $y_{i} \in Y_{i}$ and $i \in N$. Thus, a vector $b_{i}$ and a constant $c_{i}$ are determined by the system of linear equations

$$
\sum_{j} q_{i j} b_{j}^{\prime} C_{j i} C_{i i}^{-1}=G_{i}^{\prime} C_{i i}^{-1} \text { and } \sum_{j} q_{i j} c_{j}=E\left[\theta_{i}\right] \text { for } i \in N
$$

This is reduced to the following form:

$$
\sum_{j} q_{i j} C_{i j} b_{j}=G_{i} \text { and } \sum_{j} q_{i j} c_{j}=E\left[\theta_{i}\right] \text { for } i \in N .
$$

\footnotetext{
${ }^{7}$ Let $X=\left(X_{1}, X_{2}\right)$ be a random vector whose distribution is multivariate normal. Let $\mu_{i}=E X_{i}$ and $C_{i j}=$ $\operatorname{Cov}\left(X_{i}, X_{j}\right)$ for $i, j=1,2$. Then, $E\left[X_{2} \mid X_{1}\right]=\mu_{2}+C_{21} C_{11}^{-1}\left(X_{1}-\mu_{1}\right)$.
} 


\section{Information structures and efficiency}

In order to study the relationship between information structures and efficiency, we propose the following steps of analysis. For the first step, construct a model of an organization using a Bayesian potential game, which is not only less restrictive than using a team but also not uncommon as discussed in Section 2. For the second step, consider information structures $\eta$ and $\eta^{\prime}$, and obtain the corresponding Bayesian Nash equilibria using the results in the previous section. For the third step, compute the sum of ex ante expected payoffs of all the players for each information structure and compare them. If the sum for $\eta$ is greater than that for $\eta^{\prime}$, then we conclude that $\eta$ is better than $\eta^{\prime}$.

For example, consider a two-player, symmetric, quadratic Bayesian potential game:

$$
\begin{aligned}
& u_{1}(a, \omega)=-a_{1}^{2}+2 \rho a_{1} a_{2}+2 \theta_{1}(\omega) a_{1}-\alpha a_{2}^{2}+2 \beta \theta_{2}(\omega) a_{2}+f_{1}(\omega), \\
& u_{2}(a, \omega)=-a_{2}^{2}+2 \rho a_{1} a_{2}+2 \theta_{2}(\omega) a_{2}-\alpha a_{1}^{2}+2 \beta \theta_{1}(\omega) a_{1}+f_{2}(\omega)
\end{aligned}
$$

where $\theta_{1}, \theta_{2}, \eta_{1}$, and $\eta_{2}$ are jointly normally distributed and $|\rho|<1$. Note that if $\alpha=\beta=1$ then players have the same payoff functions (except the term $f_{i}(\omega)$ ) and it is a team. By Lemma 6 , $(\mathbf{u}, \eta)$ has a Bayesian potential function

$$
\begin{aligned}
v(a, \omega) & =-\left[\begin{array}{ll}
a_{1} & a_{2}
\end{array}\right]\left[\begin{array}{cc}
1 & -\rho \\
-\rho & 1
\end{array}\right]\left[\begin{array}{l}
a_{1} \\
a_{2}
\end{array}\right]+2\left[\begin{array}{ll}
\theta_{1}(\omega) & \theta_{2}(\omega)
\end{array}\right]\left[\begin{array}{l}
a_{1} \\
a_{2}
\end{array}\right] \\
& =-a_{1}^{2}-a_{2}^{2}+2 \rho a_{1} a_{2}+2 \theta_{1}(\omega) a_{1}+2 \theta_{2}(\omega) a_{2} .
\end{aligned}
$$

Note that $v(\cdot, \omega): A \rightarrow \mathbb{R}$ is concave because $|\rho|<1$. The following games conform to the above formulation (with suitable normalization).

- A model of a firm studied by Crémer (1990) has the following payoff functions

$$
u_{1}(a, \omega)=u_{2}(a, \omega)=\theta_{0}(\omega)\left(a_{1}+a_{2}\right)-\frac{B\left(a_{1}+a_{2}\right)^{2}+C\left(a_{1}-a_{2}\right)^{2}}{2}
$$

where $B, C>0$ are constants. Note that, for $i=1,2$,

$$
\frac{\partial^{2} u_{i}(a, \omega)}{\partial a_{1} \partial a_{2}}=C-B
$$

Thus, if $C>B$ then the game exhibits strategic complementarity and if $B>C$ then it exhibits strategic substitutability.

- A model of Cournot duopoly with a linear demand function has the following payoff functions

$$
\begin{aligned}
& u_{1}(a, \omega)=\left(\theta_{0}(\omega)-a_{1}-a_{2}\right) a_{1}-c_{1}(\theta) a_{1}, \\
& u_{2}(a, \omega)=\left(\theta_{0}(\omega)-a_{1}-a_{2}\right) a_{2}-c_{2}(\theta) a_{2}
\end{aligned}
$$

where $\theta_{0}(\omega)$ is a parameter for the demand structure and $c_{i}(\theta)$ is a marginal cost for firm $i=1,2$.

- A two-player version of a coordination game studied by Morris and Shin (2002) has the following payoff functions ${ }^{8}$

$$
\begin{aligned}
& u_{1}(a, \omega)=-\lambda\left(a_{1}-a_{2}\right)^{2}-(1-\lambda)\left(a_{1}-\theta_{0}(\omega)\right)^{2}, \\
& u_{2}(a, \omega)=-\lambda\left(a_{1}-a_{2}\right)^{2}-(1-\lambda)\left(a_{2}-\theta_{0}(\omega)\right)^{2}
\end{aligned}
$$

${ }^{8}$ Morris and Shin (2002) assumed a continuum of players. This game will be discussed in the next section again. 
where $0 \leq \lambda \leq 1$ is a constant. In this game, player $i$ 's best response is his expected value of a weighted average of the opponent's action $\sigma_{j}\left(\eta_{j}\right)$ and the unknown parameter $\theta_{0}(\omega)$, i.e., $E\left[\lambda \sigma_{j}\left(\eta_{j}\right)+(1-\lambda) \theta_{0}(\omega) \mid \eta_{i}(\omega)=y_{i}\right]$ for all $y_{i} \in Y_{i}$.

Using Theorem 4, we shall obtain a Bayesian Nash equilibrium in a closed form. For the parameters of the joint normal distribution of $\left(\theta_{1}, \theta_{2}, \eta_{1}, \eta_{2}\right)$, we assume $E\left[\theta_{1}\right]=E\left[\theta_{2}\right]=E\left[\eta_{1}\right]=$ $E\left[\eta_{2}\right]=0$ and the following symmetric covariance structure:

$$
C_{11}=C_{22}=C, C_{12}=C_{21}=D, \operatorname{Cov}\left(\eta_{1}, \theta_{1}\right)=\operatorname{Cov}\left(\eta_{2}, \theta_{2}\right)=G
$$

Plugging the above into (4), solving it for $b_{i}$ and $c_{i}$, and plugging $b_{i}$ and $c_{i}$ into (3), we have the unique Bayesian Nash equilibrium

$$
\sigma_{i}^{*}\left(\eta_{i}\right)=G^{\prime}(C-\rho D)^{-1} \eta_{i}
$$

for $i=1,2$. We evaluate welfare in terms of the sum of ex ante expected payoffs:

$$
\begin{aligned}
W\left(\sigma^{*}\right)= & E\left[u_{1}\left(\sigma^{*}(\eta), \omega\right)\right]+E\left[u_{2}\left(\sigma^{*}(\eta), \omega\right)\right] \\
= & -(1+\alpha) E \sigma_{1}^{2}-(1+\alpha) E \sigma_{2}^{2}+4 \rho E \sigma_{1} \sigma_{2} \\
& +2(1+\beta) E \theta_{1} \sigma_{1}+2(1+\beta) E \theta_{2} \sigma_{2}+E f_{1}+E f_{2} \\
= & -2(1+\alpha) G^{\prime}(C-\rho D)^{-1} C(C-\rho D)^{-1} G+4 \rho G^{\prime}(C-\rho D)^{-1} D(C-\rho D)^{-1} G \\
& +4(1+\beta) G^{\prime}(C-\rho D)^{-1} G+E f_{1}+E f_{2} \\
= & 2(1-\alpha) G^{\prime}(C-\rho D)^{-1} C(C-\rho D)^{-1} G+4 \beta G^{\prime}(C-\rho D)^{-1} G+f
\end{aligned}
$$

where $f=E f_{1}+E f_{2}$. Note that, in general, a Bayesian Nash equilibrium $\sigma^{*}$ does not maximize $W$ unless both players have the same payoff functions or independent payoff functions.

We study comparative statics on $W\left(\sigma^{*}\right)$ with respect to information structures. To do so, we consider a more specific information structure given by

$$
\theta_{1}=\theta_{2}=\theta, \eta_{1}=\theta+\varepsilon_{0}+\varepsilon_{1}, \eta_{2}=\theta+\varepsilon_{0}+\varepsilon_{2}
$$

where $\theta, \varepsilon_{0}, \varepsilon_{1}$, and $\varepsilon_{2}$ are independently and normally distributed with

$$
\begin{aligned}
E[\theta] & =E\left[\varepsilon_{0}\right]=E\left[\varepsilon_{1}\right]=E\left[\varepsilon_{2}\right]=0, \\
\operatorname{Var}[\theta] & =\xi, \operatorname{Var}\left[\varepsilon_{0}\right]=\phi, \operatorname{Var}\left[\varepsilon_{1}\right]=\operatorname{Var}\left[\varepsilon_{2}\right]=\psi .
\end{aligned}
$$

Then, $C=\xi+\phi+\psi, D=\xi+\phi$, and $G=\xi$. We will fix $\xi>0$ throughout and regard $(\phi, \psi) \in \mathbb{R}_{+}^{2}$ as a parameter for an information structure $\left(\eta_{1}, \eta_{2}\right)$. Note that if $(\phi, \psi)=(0,0)$ then $\theta$ is common knowledge. Plugging the above into (5) and (6), we have

$$
\begin{aligned}
\sigma_{i}^{*}\left(\eta_{i}\right) & =\frac{\xi}{(1-\rho)(\xi+\phi)+\psi} \eta_{i} \text { for } i=1,2, \\
W\left(\sigma^{*}\right) & =F(\phi, \psi)=\frac{2(1-\alpha)(\xi+\phi+\psi) \xi^{2}}{((1-\rho)(\xi+\phi)+\psi)^{2}}+\frac{4 \beta \xi^{2}}{(1-\rho)(\xi+\phi)+\psi}+f .
\end{aligned}
$$

We first compare welfare under common knowledge of $\theta$ and that under other information structures.

Proposition 1 If $\alpha \leq 1$ and $\beta \geq 0$, then $F(0,0) \geq F(\phi, \psi)$. If $\alpha \geq 1$ and $\beta \leq 0$, then $F(0,0) \leq$ $F(\phi, \psi)$. If $\alpha=1$ and $\beta=0$, then $F(0,0)=F(\phi, \psi)$. 
Proof. Remember that $|\rho|<1$ is assumed. Rewrite

$$
\begin{aligned}
F(\phi, \psi) & =\frac{2(1-\alpha)(\xi+\phi+\psi) \xi^{2}}{(1-\rho)^{2}(\xi+\phi+\psi /(1-\rho))^{2}}+\frac{4 \beta \xi^{2}}{(1-\rho)(\xi+\phi+\psi /(1-\rho))}+f \\
& =A \frac{\xi+x}{(\xi+y)^{2}}+B \frac{1}{\xi+y}+f
\end{aligned}
$$

where $A=\frac{2(1-\alpha) \xi^{2}}{(1-\rho)^{2}}, B=\frac{4 \beta \xi^{2}}{1-\rho}, x=\phi+\psi \geq 0$, and $y=\phi+\psi /(1-\rho) \geq 0$. Then,

$$
\begin{aligned}
F(\phi, \psi)-F(0,0) & =A\left(\frac{\xi+x}{(\xi+y)^{2}}-\frac{1}{\xi}\right)+B\left(\frac{1}{\xi+y}-\frac{1}{\xi}\right) \\
& =-A \frac{(2 y-x) \xi+y^{2}}{\xi(\xi+y)^{2}}-B \frac{y}{\xi(\xi+y)}
\end{aligned}
$$

Note that $2 y-x=\phi+(1+\rho) \psi /(1-\rho) \geq 0$. Since $A$ is a positive constant times $1-\alpha$ and $B$ is a positive constant times $\beta$, this proposition is true.

This proposition asserts that common knowledge of $\theta$ results in the highest efficiency if $\alpha \leq 1$ and $\beta \geq 0$, whereas it results in the lowest efficiency if $\alpha \geq 1$ and $\beta \leq 0$. It should be noted that $F(0,0) \geq F(\phi, \psi)$ does not imply that $F(\phi, \psi)$ is decreasing in $(\phi, \psi)$ as we will see in Proposition 3 .

Crémer (1990) compared two information structures in a team: shared knowledge and diversified knowledge. Shared knowledge is an information structure $(\phi, \psi)=(c, 0)$ where players have the same information. Diversified knowledge is an information structure $(\phi, \psi)=(0, c)$ where players have conditionally independent information. Crémer (1990) showed that shared knowledge is better than diversified knowledge in a team if and only if a team exhibits strategic complementarity, i.e., $\rho \geq 0 .{ }^{9}$ We obtain the same conclusion if $\alpha \leq 1$ and $\beta \geq 0$, but the opposite conclusion if $\alpha \geq 1$ and $\beta \leq 0$.

Proposition 2 Let $c>0$ and $(\alpha, \beta) \neq(1,0)$. Suppose that $\alpha \leq 1$ and $\beta \geq 0$. Then, $F(c, 0) \geq$ $F(0, c)$ if and only if $\rho \geq 0$. Suppose that $\alpha \geq 1$ and $\beta \leq 0$. Then, $F(c, 0) \geq F(0, c)$ if and only if $\rho \leq 0$.

Proof. By calculation, we have

$$
\begin{aligned}
F(c, 0)-F(0, c)= & \frac{2(1-\alpha)(\xi+c) \xi^{2}}{((1-\rho)(\xi+c))^{2}}+\frac{4 \beta \xi^{2}}{(1-\rho)(\xi+c)} \\
& -\frac{2(1-\alpha)(\xi+c) \xi^{2}}{((1-\rho) \xi+c)^{2}}-\frac{4 \beta \xi^{2}}{(1-\rho) \xi+c} \\
= & 2(1-\alpha)(\xi+c) \xi^{2} \frac{((1-\rho) \xi+c)^{2}-((1-\rho)(\xi+c))^{2}}{((1-\rho)(\xi+c))^{2}((1-\rho) \xi+c)^{2}} \\
& +4 \beta \xi^{2} \frac{((1-\rho) \xi+c)-((1-\rho)(\xi+c))}{((1-\rho)(\xi+c))((1-\rho) \xi+c)} .
\end{aligned}
$$

Note that $(1-\rho) \xi+c \geq(1-\rho)(\xi+c)$ if and only if $\rho \geq 0$. Thus, if $1-\alpha \geq 0$ and $\beta \geq 0$, $F(c, 0)-F(0, c) \geq 0$ if and only if $\rho \geq 0$, and if $1-\alpha \leq 0$ and $\beta \leq 0, F(c, 0)-F(0, c) \leq 0$ if and only if $\rho \leq 0$.

\footnotetext{
${ }^{9}$ Prat (1996) obtained the same result for teams dropping the assumptions of quadratic payoffs and normally distributed signals.
} 
Note that if the game is a team, then $\alpha=\beta=1$, satisfying the former assumption $\alpha \leq 1$ and $\beta \geq 0$, which is the result of Crémer (1990).

Morris and Shin (2002) studied information structures where private signals consist of public information and private information. They showed that more private information always increase efficiency, whereas more public information may decrease efficiency. We have a similar claim in our setup when $\alpha \leq 1$ and $\beta \geq 0$.

Proposition 3 Let $(\alpha, \beta) \neq(1,0)$. Suppose that $\alpha \leq 1$ and $\beta \geq 0$. Then,

$$
\frac{\partial}{\partial \psi} F(\phi, \psi) \leq 0 \text { and } \frac{\partial}{\partial \phi} F(\phi, \psi) \leq 0 \Leftrightarrow \frac{(1-\alpha)(2 \rho-1)-2 \beta(1-\rho)}{(1-\rho)((1-\alpha)+2 \beta(1-\rho))} \leq \frac{\xi+\phi}{\psi} .
$$

Suppose that $\alpha \geq 1$ and $\beta \leq 0$. Then,

$$
\frac{\partial}{\partial \psi} F(\phi, \psi) \geq 0 \text { and } \frac{\partial}{\partial \phi} F(\phi, \psi) \geq 0 \Leftrightarrow \frac{(1-\alpha)(2 \rho-1)-2 \beta(1-\rho)}{(1-\rho)((1-\alpha)+2 \beta(1-\rho))} \leq \frac{\xi+\phi}{\psi} .
$$

Proof. Immediate from the following calculation:

$$
\begin{array}{r}
\frac{\partial}{\partial \psi} F(\phi, \psi)=-2(1-\alpha) \xi^{2} \frac{(1+\rho)(\xi+\phi)+\psi}{((1-\rho)(\xi+\phi)+\psi)^{3}}-\frac{4 \beta \xi^{2}}{((1-\rho)(\xi+\phi)+\psi)^{2}}, \\
\frac{\partial}{\partial \phi} F(\phi, \psi)=\frac{2 \xi^{2}}{((1-\rho)(\xi+\phi)+\psi)^{3}} \times\left[-\left((1-\alpha)(1-\rho)+2 \beta(1-\rho)^{2}\right)(\xi+\phi)\right. \\
+((1-\alpha)(2 \rho-1)-2 \beta(1-\rho)) \psi] .
\end{array}
$$

Increase in the variance of public noise $\phi$ corresponds to decrease in the accuracy of public information, and increase in the variance of private noise $\psi$ corresponds to decrease in the accuracy of private information. Given this interpretation, the claim of the above proposition when $\alpha \leq 1$ and $\beta \geq 0$ is similar to that of Morris and Shin (2002).

\section{Bayesian best-response potential games}

An analysis of a weighted Bayesian potential game is reduced to team decision problems. This is owing to the fact that the equilibrium set coincides with that of a team. Conversely, it is worth asking what class of Bayesian games have the same equilibrium set as that of a team.

For games with complete information, Morris and Ui (2004) studied such a class of games. A game $\mathbf{g}$ is said to be best-response equivalent to $\mathbf{g}^{\prime}$ if, for each $i \in N$,

$$
\arg \max _{a_{i} \in A_{i}} \int g_{i}\left(a_{i}, a_{-i}\right) d \lambda_{i}\left(a_{-i}\right)=\arg \max _{a_{i} \in A_{i}} \int g_{i}^{\prime}\left(a_{i}, a_{-i}\right) d \lambda_{i}\left(a_{-i}\right)
$$

for any probability measure $\lambda_{i}$ on $A_{-i}$ such that the integrals exist. Morris and Ui (2004) considered a game which is best-response equivalent to an identical interest game, and called the game a best-response potential game. They showed that if $v$ is concave then a class of best-response potential games are strictly larger than that of weighted potential games.

We introduce the "Bayesian" version of best-response potential games. We say that $(\mathbf{u}, \eta)$ is best-response equivalent to $\left(\mathbf{u}^{\prime}, \eta\right)$ if, for each $i \in N$,

$$
\arg \max _{a_{i} \in A_{i}} E\left[u_{i}\left(\left(a_{i}, \sigma_{-i}\left(\eta_{-i}\right)\right), \omega\right) \mid \eta_{i}(\omega)=y_{i}\right]=\arg \max _{a_{i} \in A_{i}} E\left[u_{i}^{\prime}\left(\left(a_{i}, \sigma_{-i}\left(\eta_{-i}\right)\right), \omega\right) \mid \eta_{i}(\omega)=y_{i}\right]
$$


for all $\sigma_{-i} \in \Sigma_{-i}$ and $y_{i} \in Y_{i}$ such that the interim expected payoffs exist. We consider a Bayesian game which is best-response equivalent to a Bayesian identical interest game, and call the game a Bayesian best-response potential game. A weighted Bayesian potential game is a Bayesian best-response potential game as we have seen in Section 3. By the definition of Bayesian Nash equilibria, the equilibrium set of a Bayesian best-response potential game coincides with that of the Bayesian identical interest game.

Definition 3 A Bayesian game $(\mathbf{u}, \eta)$ is a Bayesian best-response potential game and a function $v: A \times \Omega \rightarrow \mathbb{R}$ is a Bayesian best-response potential function if $(\mathbf{u}, \eta)$ is best-response equivalent to a Bayesian identical interest game $(\mathbf{v}, \eta)$ such that $\mathbf{v}=\left(v_{i}\right)_{i \in N}$ and $v_{i}=v$ for all $i \in N$.

Lemma 7 For a Bayesian best-response potential game $(\mathbf{u}, \eta)$ with a Bayesian best-response potential function $v$, let $(\mathbf{v}, \eta)$ be a Bayesian identical interest game such that $\mathbf{v}=\left(v_{i}\right)_{i \in N}$ and $v_{i}=v$ for all $i \in N$. Then, the set of Bayesian Nash equilibria of $(\mathbf{u}, \eta)$ coincides with that of $(\mathbf{v}, \eta)$.

The above lemma implies that the results in Section 4 hold even if "weighted Bayesian potential" is replaced by "Bayesian best-response potential." Thus, we are interested in Bayesian best-response potential games with concave Bayesian best-response potential functions. The following theorem characterizes such a class of Bayesian best-response potential games.

Theorem 5 Let $(\mathbf{u}, \eta)$ be a Bayesian game. Let $v: A \times \Omega \rightarrow \mathbb{R}$ be continuously differentiable and concave with respect to $a \in A$. Suppose that, for each $i \in N$, there exists a function $w_{i}: A_{i} \times Y_{i} \rightarrow$ $\mathbb{R}_{++}$such that

$$
\frac{\partial u_{i}(a, \omega)}{\partial a_{i}}=w_{i}\left(a_{i}, \eta_{i}(\omega)\right) \frac{\partial v(a, \omega)}{\partial a_{i}}
$$

for all $a \in A$ and $\omega \in \Omega$. Suppose further that $\sup _{a_{i} \in(\alpha, \beta), y_{i} \in Y_{i}} w_{i}\left(a_{i}, y_{i}\right)$ exists for any interval $(\alpha, \beta) \subseteq A_{i}$. Then, $(\mathbf{u}, \eta)$ is a Bayesian best-response potential game with a Bayesian best-response potential function $v$.

Before proving the theorem, let us discuss a simple but useful application to robustness of equilibrium sets. Let $(\mathbf{u}, \eta)$ be a Bayesian potential game with a Bayesian potential function $v$ that is continuously differentiable and concave with respect to $a \in A$. Let $\left(\mathbf{u}^{\prime}, \eta\right)$ be another Bayesian game such that

$$
u_{i}^{\prime}(a, \omega)=w_{i}\left(\eta_{i}(\omega)\right) u_{i}(a, \omega)
$$

for all $a \in A, \omega \in \Omega$, and $i \in N$ where $w_{i}: Y_{i} \rightarrow \mathbb{R}_{++}$is a $\eta_{i}$-measurable random variable taking a strictly positive value. By Theorem $5,\left(\mathbf{u}^{\prime}, \eta\right)$ is a Bayesian best-response potential game with a Bayesian best-response potential function $v$. Thus, by Lemma 7 , the set of Bayesian Nash equilibria of $\left(\mathbf{u}^{\prime}, \eta\right)$ coincides with that of $(\mathbf{u}, \eta)$. In other words, the set of Bayesian Nash equilibria of $(\mathbf{u}, \eta)$ is robust to random disturbances of payoff functions of the form (8).

To prove the theorem, we use the following lemma.

Lemma 8 For each $\sigma_{-i} \in \Sigma_{-i}$ and $y_{i} \in Y_{i}$ such that the interim expected payoffs exist,

$$
\begin{aligned}
\frac{\partial}{\partial a_{i}} E\left[v\left(\left(a_{i}, \sigma_{-i}\left(\eta_{-i}\right)\right), \omega\right) \mid \eta_{i}(\omega)\right. & \left.=y_{i}\right]=E\left[\frac{\partial}{\partial a_{i}} v\left(\left(a_{i}, \sigma_{-i}\left(\eta_{-i}\right)\right), \omega\right) \mid \eta_{i}(\omega)=y_{i}\right], \\
\frac{\partial}{\partial a_{i}} E\left[u_{i}\left(\left(a_{i}, \sigma_{-i}\left(\eta_{-i}\right)\right), \omega\right) \mid \eta_{i}(\omega)\right. & \left.=y_{i}\right]=E\left[\frac{\partial}{\partial a_{i}} u_{i}\left(\left(a_{i}, \sigma_{-i}\left(\eta_{-i}\right)\right), \omega\right) \mid \eta_{i}(\omega)=y_{i}\right] .
\end{aligned}
$$


Proof. Since $v(a, \omega)$ is concave in $a \in A, v\left(\left(x+d, \sigma_{-i}\left(\eta_{-i}\right)\right), \omega\right)-v\left(\left(x, \sigma_{-i}\left(\eta_{-i}\right)\right), \omega\right)$ is concave in $d$, and thus $\left[v\left(\left(x+d, \sigma_{-i}\left(\eta_{-i}\right)\right), \omega\right)-v\left(\left(x, \sigma_{-i}\left(\eta_{-i}\right)\right), \omega\right)\right] / d$ increases to $\partial v(a, \omega) /\left.\partial a_{i}\right|_{a=\left(x, \sigma_{-i}\left(\eta_{-i}\right)\right)}$ as $d \rightarrow+0$. Thus, by the monotone convergence theorem, we must have (9). The concavity of $v$ also implies that $\partial v(a, \omega) / \partial a_{i}$ is decreasing in $a_{i} \in A_{i}$. Thus, for any $\alpha, \beta, x \in A_{i}$ with $\alpha<x<\beta$, (7) implies that

$$
\left|\frac{\partial u_{i}(a, \omega)}{\partial a_{i}}\right|_{a_{i}=x} \mid \leq \bar{w}_{i}\left(\left.\left|\frac{\partial v(a, \omega)}{\partial a_{i}}\right|_{a_{i}=\alpha}|+| \frac{\partial v(a, \omega)}{\partial a_{i}}\right|_{a_{i}=\beta} \mid\right)
$$

where $\bar{w}_{i}=\sup _{a_{i} \in(\alpha, \beta), y_{i} \in Y_{i}} w_{i}\left(a_{i}, y_{i}\right)$. Note that, for each $x \in(\alpha, \beta)$ and $d \in(0, \beta-x)$, there exists $\gamma \in[x, x+d] \subseteq(\alpha, \beta)$ by the mean-value theorem such that

$$
\frac{u_{i}\left(\left(x+d, a_{-i}\right), \omega\right)-u_{i}\left(\left(x, a_{-i}\right), \omega\right)}{d}=\left.\frac{\partial u_{i}(a, \omega)}{\partial a_{i}}\right|_{a_{i}=\gamma} .
$$

Thus,

$$
\begin{aligned}
& \left|\frac{u_{i}\left(\left(x+d, \sigma_{-i}\left(\eta_{-i}\right)\right), \omega\right)-u_{i}\left(\left(x, \sigma_{-i}\left(\eta_{-i}\right)\right), \omega\right)}{d}\right| \\
& \quad \leq \bar{w}_{i}\left(\left.\left|\frac{\partial v(a, \omega)}{\partial a_{i}}\right|_{a=\left(\alpha, \sigma_{-i}\left(\eta_{-i}\right)\right)}|+| \frac{\partial v(a, \omega)}{\partial a_{i}}\right|_{a=\left(\beta, \sigma_{-i}\left(\eta_{-i}\right)\right)} \mid\right) .
\end{aligned}
$$

Since the right-hand side is integrable with respect to $E\left[\cdot \mid \eta_{i}(\omega)=y_{i}\right]$, by the dominated convergence theorem, we must have (10).

Proof of Theorem 5. By (7), (9), and (10),

$$
\frac{\partial}{\partial a_{i}} E\left[u_{i}\left(\left(a_{i}, \sigma_{-i}\left(\eta_{-i}\right)\right), \omega\right) \mid \eta_{i}(\omega)=y_{i}\right]=w_{i}\left(a_{i}, y_{i}\right) \frac{\partial}{\partial a_{i}} E\left[v\left(\left(a_{i}, \sigma_{-i}\left(\eta_{-i}\right)\right), \omega\right) \mid \eta_{i}(\omega)=y_{i}\right] .
$$

This implies that

$$
\begin{aligned}
\operatorname{sign}\left(\frac { \partial } { \partial a _ { i } } E \left[u_{i}\left(\left(a_{i}, \sigma_{-i}\left(\eta_{-i}\right)\right), \omega\right) \mid\right.\right. & \left.\left.\eta_{i}(\omega)=y_{i}\right]\right) \\
= & \operatorname{sign}\left(\frac{\partial}{\partial a_{i}} E\left[v\left(\left(a_{i}, \sigma_{-i}\left(\eta_{-i}\right)\right), \omega\right) \mid \eta_{i}(\omega)=y_{i}\right]\right) .
\end{aligned}
$$

By the concavity of $v, \partial v(a, \omega) / \partial a_{i}$ is decreasing in $a_{i}$, and thus the both sides of $(9)$ are decreasing in $a_{i}$. Accordingly,

$$
a_{i}^{*} \in \arg \max _{a_{i} \in A_{i}} E\left[v\left(\left(a_{i}, \sigma_{-i}\left(\eta_{-i}\right)\right), \omega\right) \mid \eta_{i}(\omega)=y_{i}\right]
$$

if and only if (11) is 0 or plus for all $a_{i}<a_{i}^{*}$ and 0 or minus for all $a_{i}>a_{i}^{*}$. This is true if and only if

$$
a_{i}^{*} \in \arg \max _{a_{i} \in A_{i}} E\left[u_{i}\left(\left(a_{i}, \sigma_{-i}\left(\eta_{-i}\right)\right), \omega\right) \mid \eta_{i}(\omega)=y_{i}\right],
$$

which completes the proof.

The next corollary applies Theorem 5 to a quadratic Bayesian potential game considered in Lemma 6.

Corollary 6 Let $(\mathbf{u}, \eta)$ be a Bayesian game. Let $v: A \times \Omega \rightarrow \mathbb{R}$ be such that

$$
v(a, \omega)=-a^{\prime} Q(\omega) a+2 \theta(\omega)^{\prime} a
$$


where $Q(\omega)=\left[q_{i j}(\omega)\right]_{n \times n}$ is a positive definite matrix and $\theta(\omega)=\left[\theta_{1}(\omega), \ldots, \theta_{n}(\omega)\right]^{\prime}$ is a column vector for all $\omega \in \Omega$. Suppose that, for each $i \in N$, there exists a function $f_{i}: A_{i} \times Y_{i} \rightarrow \mathbb{R}$ such that $f_{i}\left(\cdot, y_{i}\right): A_{i} \rightarrow \mathbb{R}$ is continuously differentiable and strictly increasing for each $y_{i} \in Y_{i}$ and

$$
u_{i}(a, \omega)=f_{i}\left(a_{i}, \eta_{i}(\omega)\right)\left(-2 \sum_{j \in N} q_{i j}(\omega) a_{j}+2 \theta_{i}(\omega)\right)+2 q_{i i}(\omega) \int_{0}^{a_{i}} f_{i}\left(x, \eta_{i}(\omega)\right) d x+h_{i}\left(a_{-i}, \omega\right)
$$

for all $a \in A$ and $\omega \in \Omega$. Then, $(\mathbf{u}, \eta)$ is a Bayesian best-response potential game with a Bayesian best-response potential function $v$. Especially, if $f_{i}\left(a_{i}, a_{i}\right)=a_{i}$, then $(\mathbf{u}, \eta)$ is a quadratic Bayesian potential game given in Lemma 6.

Proof. Let $w_{i}\left(a_{i}, \eta_{i}(\omega)\right)=\partial f_{i}\left(a_{i}, \eta_{i}(\omega)\right) / \partial a_{i}>0$. Then, Theorem 5 establishes the corollary.

As an example, we consider a finite-player version of a coordination game studied by Morris and Shin (2002), which induces strategic behavior in the spirit of the "beauty contest" example of Keynes (1936). Assume that $n \geq 3$. The payoff function is given by

$$
u_{i}(a, \omega)=-(1-\lambda)\left(a_{i}-\theta_{0}(\omega)\right)^{2}-\lambda\left(L_{i}-\bar{L}\right)
$$

where $0<\lambda<1$ and

$$
L_{i}=\frac{1}{n} \sum_{i \in N}\left(a_{j}-a_{i}\right)^{2}, \bar{L}=\frac{1}{n} \sum_{i \in N} L_{i} .
$$

Then, we can calculate the following:

$$
\frac{\partial u_{i}(a, \omega)}{\partial a_{i}}=-2 c\left(a_{i}-\left(\rho \frac{\sum_{j \neq i} a_{j}}{n-1}-(1-\rho) \theta_{0}(\omega)\right)\right)
$$

where

$$
\rho=\frac{(n-1)(n-2)}{n^{2}-(3 n-2) \lambda} \lambda, c=1-\frac{3 n-2}{n^{2}} \lambda .
$$

Note that $\rho \rightarrow \lambda$ and $c \rightarrow 1$ as $n \rightarrow \infty$, which corresponds to the infinite-player case. By the first-order condition given by (13), we can find that player $i$ 's best response is a weighted sum of the conditional expectation of the opponents' actions $\sum_{j \neq i} \sigma_{j}\left(\eta_{j}\right) /(n-1)$ and that of $\theta_{0}(\omega)$, i.e.,

$$
\rho \frac{\sum_{j \neq i} E\left[\sigma_{j}\left(\eta_{j}\right) \mid \eta_{i}(\omega)=y_{i}\right]}{n-1}+(1-\rho) E\left[\theta_{0}(\omega) \mid \eta_{i}(\omega)=y_{i}\right]
$$

for all $y_{i} \in Y_{i}$. This represents the spirit of the "beauty contest" example, and Angeletos and Pavan(2007) call this game a beauty contest game. In this game, Morris and Shin (2002) studied information structures where private signals consist of public information and private information, and showed that more private information always increase efficiency, whereas more public information may decrease efficiency.

Following Morris and Shin (2002), the payoff function of the form (12) is widely discussed. However, it is an open question to provide a proper microfoundation. On the other hand, the essence of Morris and Shin (2002) lies in the best response of the form (14), not in the specific payoff function of the form (12). Thus, it is interesting and important to ask what class of Bayesian games have the best response of the form (13). Corollary 6 provides an answer to this question.

To see this, observe that, by Lemma 6 , the above game is a weighted Bayesian potential game with a weighted Bayesian potential function

$$
v(a)=-\frac{1}{1-\rho} \sum_{i \in N} a_{i}^{2}+\frac{\rho}{(n-1)(1-\rho)} \sum_{i, j \in N, i \neq j} a_{i} a_{j}+2\left(\sum_{i \in N} a_{i}\right) \theta_{0}(\omega) .
$$


Note that $\partial v / \partial a_{i}$ is equal to the left-hand side of (13) divided by $c(1-\rho)$. Now, consider the following payoff function.

$$
\begin{aligned}
u_{i}^{\prime}(a, \omega)= & f_{i}\left(a_{i}, \eta_{i}(\omega)\right)\left(\frac{-2}{1-\rho} a_{i}+\frac{2 \rho}{(n-1)(1-\rho)} \sum_{j \neq i} a_{j}+2 \theta_{0}(\omega)\right) \\
& +\frac{2}{1-\rho} \int_{0}^{a_{i}} f_{i}\left(x, \eta_{i}(\omega)\right) d x+h_{i}\left(a_{-i}, \omega\right)
\end{aligned}
$$

where $f_{i}\left(\cdot, y_{i}\right): A_{i} \rightarrow \mathbb{R}$ is a continuously differentiable, strictly increasing function for each $i \in N$ and $y_{i} \in Y_{i}$. Then, by Corollary $6,\left(\mathbf{u}^{\prime}, \eta\right)$ is a Bayesian best-response potential game with a Bayesian best-response potential function $v$, and player $i$ 's best response is a weighted average of the conditional expectation of $\sum_{j \neq i} \sigma_{j}\left(\eta_{j}\right) /(n-1)$ and that of $\theta_{0}(\omega)$. It can be shown that the game studied by Morris and Shin (2002) is a special case with

$$
\begin{aligned}
f_{i}\left(a_{i}, y_{i}\right) & =c(1-\rho) a_{i}, \\
h_{i}\left(a_{-i}, \omega\right) & =-(1-\lambda) \theta_{0}(\omega)^{2}+\frac{\lambda}{n^{2}}\left((n+1) \sum_{j \neq i} a_{j}^{2}-2\left(\sum_{j \neq i} a_{j}\right)^{2}\right) .
\end{aligned}
$$

\section{References}

[1] Angeletos, G.-M., and A. Pavan (2007), "Efficient use of information and social value of information," Econometrica 75, 1103-1142.

[2] Aoki, M. (1986), "Horizontal vs. vertical information structure of the firm," American Economic Review 76, 971-983.

[3] Aoki, M. (1995), "An evolving diversity of organizational mode and its implications for transitional economies," Journal of the Japanese and International Economies 9, 330-353.

[4] Arrow, K. J. (1985), "Informational structure of the firm," American Economic Review 75, 303-307.

[5] Arrow, K. J., and R. Radner (1979), "Allocation of resources in large teams," Econometrica 47, 361-386.

[6] Basar, T., and Y. Ho (1974), "Informational properties of the Nash solution of the two stochastic nonzero-sum games," Journal of Economic Theory 7, 370-384.

[7] Bassan, B., O. Gossner, M. Scarsini, and S. Zamir (2003), "Positive value of information in games," International Journal of Game Theory 32, 17-31.

[8] Bassan, B., M. Scarsini, and S. Zamir (1997), "I don't want to know!: Can it be rational?," Discussion Paper No. 158, Center for Rationality and Interactive Decision Theory.

[9] Bolton, B., and M. Dewatripont (1994), "The firm as a communication network," Quarterly Journal of Economics 109, 809-839.

[10] Clark, R. (1983), "Collusion and the incentives for information sharing," Bell Journal of Economics 14, 383-394. 
[11] Crémer, J. (1980), "A partial theory of the optimal organization of a bureaucracy," Bell Journal of Economics 11, 683-693.

[12] Crémer, J. (1990), "Common knowledge and the coordination of economic activities," M. Aoki, B. Gustafsson, and O. E. Williamson, eds., The firm as a nexus of treaties, 53-76, London: Sage Publishers.

[13] Crémer, J. (1993), "Corporate culture and shared knowledge," Industrial and Corporate Change 2, 351-386.

[14] Gal-Or, E. (1985), "Information sharing in oligopoly," Econometrica 53, 329-343.

[15] Gal-Or, E. (1986), "Information transmission-Cournot and Bertrand equilibria," Review of Economic Studies 53, 85-92.

[16] Geanakoplos, J., and P. Milgrom (1991), "A theory of hierarchies based on limited managerial attention," Journal of the Japanese and International Economies 5, 205-225.

[17] Gossner, O. (2000), "Comparison of information structures," Games and Economic Behavior 30, 44-63.

[18] Groves, T., and R. Radner (1972), "Allocation of resources in a team," Journal of Economic Theory 30, 44-63.

[19] Hart, O., and J. Moore (1990), "Property rights and the nature of the firm," Journal of Political Economy 98, 1119-1158.

[20] van Heumen, R., B. Peleg, S. Tijs, and P. Borm (1996), "Axiomatic characterizations of solutions for Bayesian games," Theory and Decision 40, 103-130.

[21] Itoh, H. (1987), "Information processing capacities of the firm," Journal of the Japanese and International Economies 1, 299-326.

[22] Keynes, J. M. (1936), The General Theory of Employment, Interest and Money, London: Macmillan Cambridge University Press.

[23] Krainak, J. C., J. L. Speyer, and S. I. Marcus (1982), "Static team problems-Part I: Sufficient conditions and the exponential cost criterion," IEEE Transactions on Automatic Control 27, 839-848.

[24] Levine, P., and J. P. Ponssard (1977), "The value of information in some non-zero sum games," International Journal of Game Theory 6, 221-229.

[25] Marshak, J. (1955), "Elements for a theory of teams," Management Science 1, 127-137.

[26] Marshak, J., and R. Radner (1972), Economic Theory of Teams, New Haven: Yale University Press.

[27] Monderer, D., and L. S. Shapley (1996), "Potential games," Games and Economic Behavior 14, 124-143.

[28] Morris, S., and H. S. Shin (2002), "Social value of public information," American Economic Review 92, 1521-1534. 
[29] Morris, S., and T. Ui (2004), "Best response equivalence," Games and Economic Behavior 49, 260-287.

[30] Neyman, A. (1991), "The positive value of information," Games and Economic Behavior 3, $350-355$.

[31] Neyman, A. (1997), "Correlated equilibrium and potential games," International Journal of Game Theory 26, 223-227.

[32] Prat, A. (1996), "Shared knowledge vs. diversified knowledge in teams," Journal of the Japanese and International Economies 10, 181-195.

[33] Prat, A. (2002), "Should a team be homogeneous?," European Economic Review 46, 11871207.

[34] Qian, Y., G. Roland, and C. Xu (2000), "Coordinating changes in M-form and U-form organizations," Working Paper No. 284, William Davidson Institute.

[35] Radner, R. (1961), "The evaluation of information in organizations," Proceedings of the Fourth Berkeley Symposium on Mathematical Statistics and Probability 1, 491-530.

[36] Radner, R. (1962), "Team decision problems," Annals of Mathematical Statistics 33, 857-881.

[37] Radner, R. (1992), "Hierarchy: The economics of managing," Journal of Economic Literature 30, 1382-1415.

[38] Raith, M. (1996), "A general model of information sharing in oligopoly," Journal of Economic Theory 71, 260-288.

[39] Slade, M. E. (1994), "What does an oligopoly maximize?," Journal of Industrial Economics 42, 45-61.

[40] Ui, T. (2000), "A Shapley value representation of potential games," Games and Economic Behavior 31, 121-135.

[41] Vives, X. (1984), "Duopoly information equilibrium: Cournot and Bertrand," Journal of Economic Theory 34, 71-94.

[42] Vives, X. (1988), "Aggregation of information in large Cournot markets," Econometrica 56, $851-876$.

[43] Van Zandt, T. (1999), "Decentralized information processing in the theory of organizations," M. Sertel, ed., Contemporary economic issues vol. 4: Economic design and behavior, 125-160, London: Macmillan Press. 